ESAIM: PROCEEDINGS AND SURVEYS, September 2014, Vol. 45, p. 48-57

J.-S. Dhersin, Editor

\title{
CLASSIFYING HEARTRATE BY CHANGE DETECTION AND WAVELET METHODS FOR EMERGENCY PHYSICIANS *
}

\author{
Nourddine Azzaoui ${ }^{1}$, Arnaud Guillin ${ }^{1}$, Frederic Dutheil ${ }^{2,3,4,5}$, Gil Boudet ${ }^{3}$, \\ Alain Chamoux ${ }^{4}$, Christophe Perrier ${ }^{5}$, Jeannot Schmidt ${ }^{5}$ and Pierre Raphä̈L \\ BERTRAND ${ }^{1}$
}

\begin{abstract}
Heart Rate Variability (HRV) carries a wealth of information about the physiological state and the behaviour of a living individual. Indeed, the heart rate variation is intrinsically linked to the autonomic nervous system: the parasympathetic and orthosympathetic systems. Thus, any imbalance in these two opposite systems results in a variation of the cardiac frequency modulation. This alternation between equilibrium and disequilibrium (frequency variability) is recognized as an indicator of well-being and good health. Particularly, decreased HRV is linked to stress, fatigue and decreased physical performance. The aim of this work is to exploit the heart rate signals to detect stressful situations in different populations: emergency physicians, sportsmen, animal behaviours... We introduce a methodological framework for the detection of stress and eventually well-being. Our contribution is firstly based on using Gabor wavelets to extract energies corresponding to High and Low Frequency ( $\mathrm{HF}$ and LF) bands which are linked to the parasympathetic and orthosympathetic systems. We then detect change points on these energies using the Filtered Derivative with p-value (FDpV) method. Finally, we develop a typology of cardiac activity by distinguishing homogeneous groups or state profiles sharing similar characteristics. We apply our methodology on a real dataset collected by monitoring cardiac activity of an emergency physician for 24 hours.
\end{abstract}

Résumé. La variabilité sinusale est porteuse de riches informations sur l'état physiologique et comportemental d'un individu. En effet, la variation du rythme cardiaque est intrinsèquement liée au système nerveux centrale autonome : les systèmes Parasympathique et Orthosympathique. Ainsi, tout déséquilibre dans ces deux systèmes se traduit par une variation de la modulation de la fréquence cardiaque. Cette alternance entre équilibre et déséquilibre (en l'occurrence ici une grande variabilité de la fréquence) est considérée comme un indicateur de bonne santé : une diminution de la variabilité sinusale est liée au stress, à la fatigue et à la diminution des performances physiques. Le but de ce travail est d'exploiter le rythme cardiaque pour détecter des situations de stress dans différentes populations : médecins urgentistes, sportifs amateurs, comportements animaliers... Nous élaborons une typologie de l'activité cardiaque en distinguant des groupes homogènes ou des profils d'états partageant une certaine ressemblance.

Nous appliquons ensuite notre méthodologie à un jeu de données réelles correspondant à une garde d'un médecin urgentiste.

* Research supported by grant ANR-12-BS01-0016-01 entitled "Do Well B."

${ }^{1}$ Laboratoire de Mathématiques, UMR 6620 CNRS et Université Blaise Pascal (Clermont-Ferrand 2), France.

2 School of Exercise Science, Australian Catholic University, Melbourne, Victoria, Australia

${ }^{3}$ Department of Occupational Medicine, University Hospital (CHU), G. Montpied Hospital, Clermont-Ferrand, France

${ }^{4}$ Laboratory of Metabolic Adaptations to Exercise in Physiological and Pathological Conditions EA3533, Blaise Pascal University, Clermont-Ferrand, France

${ }^{5}$ Emergency Department, University Hospital (CHU), G. Montpied Hospital, Clermont-Ferrand, France

(C) EDP Sciences, SMAI 2014 


\section{INTRODUCTION}

Technological development and advanced electronic miniaturization makes it possible to produce physiological measurement devices which are increasingly reliable and accurate. Indeed, we live on the brink of a massive development of devices and sensors able of doing measurements almost as accurate as in a specialized medical center. For a total autonomy of users, these devices should be able to be handled by the general public without the help of a healthcare professional. The users autonomy will not be insured without the development of novel algorithms and more accurate mathematical models. In this paper, we focus on the analysis of RR-intervals (Times between consecutive R peaks see Figure 1) deduced from the electrocardiogram (ECG) signals. The RR series contains a wealth of information about the health status and the internal behaviour of an individual [10]. Indeed, ECG signal analysis has come a long way since the implementation of the ambulatory monitoring by Holter at the beginning of the fifties. Recent measurement methods allow to record ECG data for healthy people over a long period of time: long distance (marathon runners), individual daily (24 hours) records, [8,11]... From these large datasets the variations of heartbeat durations were characterized in the two components of the autonomous nervous system: the parasympathetic and orthosympathetic systems.

Traditionally, these signals were analyzed by the experienced eye of cardiologists. The software solutions recently introduced provide some data summary statistics or possibly indications about the physiological state of a user. The most popular is the instantaneous average frequency which is displayed by runners' watches or integrated in the latest generation smartphones. This quantity gives poor indications about daily activities of an individual and does not summarize all the relevant information. Note that heartbeat data of healthy subjects display large variations and clustering against a regular heart rate for individuals with serious diseases.

Moreover, cardiologists are interested in the study of this signal in two frequency bands: the orthosympathetic and parasympathetic bands, i.e., the low frequency $L F=(0.04 \mathrm{~Hz}, 0.15 \mathrm{~Hz})$ and the high frequency $H F=$ $(0.15 \mathrm{~Hz}, 0.5 \mathrm{~Hz})$ bands, respectively. The definition of these bands is the outcome research work of the Task force [7], and is based on the fact that the energy contained inside these bands would be a relevant indicator on the level of stress of an individual. Indeed, for the heart rate, the parasympathetic system is often compared to the brake while the orthosympathetic system would be an accelerator [13]. At rest there is a permanent braking effect on the heart rate. Any solicitation of the cardiovascular system, any activity initially produces a reduction of parasympathetic brake followed by a gradual involvement of the sympathetic system. Thus, in the field of physiology these data are crucial to investigate parameters such as the level of vigilance, general perceived stress or specific stress induced by physical activity.

Fractal models have been used in cardiology by $[12,14]$ who used multifractal spectrums to discriminate between individuals who experienced heart trouble, and those who did not. However, this tool has some shortcomings as it requires huge samples and is then inappropriate for studying phenomena occurring at a resolution lower than the daily time intervals, such as intra-day variations of parasympathetic and orthosympathetic systems.

Wavelet-based methods have been used in biostatistics for uterine Electromyography (EMG) signal analysis [9]. They used wavelet techniques as a classification tool by considering the studied EMG signal as a homogeneous process. The main criticism of this work is, on one hand the use of discrete wavelet decomposition, i.e., a frequency decomposition on a dyadic wavelet basis. On the other hand, the choice of frequency bands without reference to the studied biological phenomenon. In our work, the choice of the frequency band is justified by biological considerations about human heart rate. The continuous Gabor wavelets are then fitted inside these bands.

In the rest of this paper, our plan will be as follows: In Section 1, we describe the probabilistic model and the statistical tools. In Section 2, we process a real dataset corresponding to emergency physicians with these tools. Section 3 gives some conclusion and state the direction of a future research. 


\section{Models and methodological approach}

In Subsection 1.1, we give a brief description of the mathematical model by considering the heart rate activity as a locally stationary process. We present a brief description of the Filtered Derivative with p-Value $(\mathrm{FDpV})$ method enabling the detection of change points. We also detail how we use the Gabor wavelets to extract energies corresponding to the sympathetic and the parasympathetic systems; i.e. the HF and LF bands. Subsection 1.2 contains a description on how we construct the discriminating variables from change points detected on RR-signals, HF and the LF energies. We also present the classification tools used for extracting homogeneous heart rate profiles.

\subsection{The RR-signals as a locally stationary process}

Even in laboratory conditions, where a precise protocol is followed and the environment is well controlled, heartbeat durations are a random series. Furthermore in real life situation, both environment condition and stress levels vary. In particular, this is the case for emergency physicians.

Let us recall that the duration of each heartbeat can be obtained as an RR-interval, which is the time interval between two successive R-waves registered by the ECG, see Fig.1.

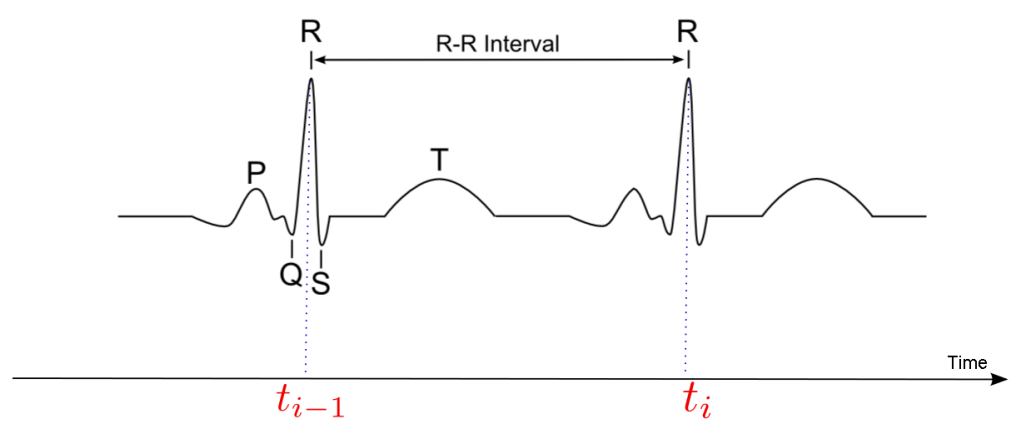

FigURE 1. Illustration of RR signal measurement

Next, we can precisely measure the time of each maximum of R-wave, and we denote it by $t_{i}$. Then, the duration of the $i^{t} h$ heartbeat is exactly $X_{i}=t_{i}-t_{i-1}$. The instantaneous heart rate (HR) is provided by the equation $H R=60 / R R$, where $R R=X\left(t_{i}\right)$ is measured in seconds, $H R$ is measured in beats per minute. For biological reasons, the human HR is within the interval $[20,250]$ bpm (beats per minute), i.e., $X(t)$ belongs to the RR interval $60 / 250 s$. $<X(t)<60 / 20 s$. In real life situations, it is quite natural to consider nychthemeron activities as a sequence of more or less active periods (sleep, rest, stress,...). This leads to modeling RR-series $X\left(t_{i}\right)$ by locally stationary processes for both time and frequency domains.

Therefore, we assume that the signal is the sum of a piecewise constant function and a Gaussian process, centered and locally stationary. We then have the following representation:

$$
X(t)=\mu(t)+\int_{\mathbb{R}} e^{i t \xi} \sqrt{f(t, \xi)} d B(\xi), \text { for all } t \in \mathbb{R},
$$

where

- $B(\xi)$ is a "well-balanced" Wiener measure, such that $X(t)$ is a real number, for all $t \in \mathbb{R}$, see e.g. [18] or [3] [2], for a precise definition.

- The map $\xi \mapsto f(t, \xi)$ is an even and positive function, called spectral density. We assume that the spectral density is piecewise constant with respect to time, namely there exists a partition $\tau_{1}, \ldots, \tau_{K}$ such that $f(t, \xi)=f_{k}(\xi)$ for $t \in\left[\tau_{i}, \tau_{i+1}[\right.$. 
- the function $t \mapsto \mu(t)$ is also piecewise constant for another partition $\tilde{\tau}_{1}, \ldots, \tilde{\tau}_{L}$ with $\mu(t)=\mu_{\ell}$ if $t \in\left[\tilde{\tau}_{\ell}, \tilde{\tau}_{\ell+1}[\right.$.

The first step of data processing treated in this work will be the detection of instants where the parameters of the process $X(t)$ will present an abrupt change in either time or frequency components.

Change points detection: the FDpV method

Change points detection is well studied in literature, we cite among others [5,6]. The classical proposed solutions are usually based on penalized least square techniques which suffer from memory complexity and time consuming calculations. To overcome this problem Bertrand et al. [6] introduced a faster technique. The main idea of the FDpV method is based on two steps, the first consists in detecting changes without worrying about false detections, the second one consist in performing statistical test to remove false change points. The FDpV method has a theoretical complexity of order $\mathcal{O}(n)$ while classical approaches are of order $\mathcal{O}\left(n^{2}\right)$. This technique have been optimized and coded with a java software and is able of making real-time detection.

Time-frequency analysis in HF and LF bands

According to recommendations of the Task force [7], we use the following notations :

- $\left[\omega_{1}, \omega_{2}\right]=(0.04 \mathrm{~Hz}, 0.15 \mathrm{~Hz})$ denotes the orthosympathetic frequency band;

- $\left[\omega_{2}, \omega_{3}\right]=(0.15 \mathrm{~Hz}, 0.5 \mathrm{~Hz})$ denotes the parasympathetic band

In order to efficiently extract energies corresponding to HF and LF bands we use techniques presented in [1] or [4]. Indeed they have introduced a theoretical study of the wavelet coefficients for stationary (or with stationary increment) centered Gaussian processes, i.e., for X given by (1) with $\mu(t)=0$. They have also generalized this result to locally stationary Gaussian processes. We give here a brief description of their technique: using a suitable wavelet, they extract the energies associated with LF and HF bands and localized around the time $b$. This is measured by the modulus of the complex wavelet coefficients $\left|W_{i}(b)\right|^{2}$ for $i=1,2$, with

$$
W_{i}(b)=\int_{\mathbb{R}} \psi_{i}(\mathrm{t}-\mathrm{b}) \mathrm{X}(\mathrm{t}) d t
$$

where $\psi_{1}$ and $\psi_{2}$ are suitable wavelets chosen with disjoint frequency supports corresponding to the LF and HF bands. These wavelet coefficients are then computed at each second, i.e., the difference between two consecutive values for $b$ is equal to 1 second.

Then we consider the change point problem of the mean of the multivariate time series $Z_{1}(b), Z_{2}(b)$ where

$$
Z_{1}(b)=\log \left(\left|W_{1}(b)\right|^{2}\right), \quad \text { and } \quad Z_{2}(b)=\log \left(\left|W_{2}(b)\right|^{2}\right),
$$

and we use a simple method well suited for big datasets, that is the Filtered Derivative with p-value (FDpV) method $[6,16]$.

How to choose the wavelets $\psi_{1}$ and $\psi_{2}$ ?

In the idealistic case, we would use two filters $\psi_{1}$ and $\psi_{2}$ with compact support, the Fourier transforms of which have support inside the orthosympathetic and parasympathetic bands. Unfortunately, there is no function $\psi$ with compact time domain support and compact frequency support, see for instance [17, Th 2.6 p.34] . Therefore, the best we can do is to choose between a filter with a compact frequency support and a filter with a compact time domain support. The first choice is well suited for stationary models [2]. The price to pay for the compactness of the time domain support is the loss in the compactness of the frequency support. To evaluate the effect of the compactness loss, Ayache and Bertrand [1] have introduced the notion of $\rho$ pseudo support. This means that the quantity $1-\rho$ evaluate the energy loss if we force the time support to 
be compact ${ }^{1}$. The idea is then to adjust the pseudo support inside a specified frequency band where $\rho$ is close to 1 . We have proposed a generic method permitting to find such supports by scaling and modulation [3]. For the sake of readability, let us recall the following proposition:

Proposition 1.1. Let $\psi$ be a filter with compact support $\left[L_{1}, L_{2}\right]$ and a frequency $\rho$ pseudo support $\left[\Lambda_{1}, \Lambda_{2}\right]$. Let us consider an arbitrary frequency band $\left[\omega_{1}, \omega_{2}\right]$ and denote,

$$
\lambda=\frac{\omega_{2}-\omega_{1}}{\Lambda_{2}-\Lambda_{1}}, \quad \eta=\frac{\omega_{1}+\omega_{2}}{2}-\left(\omega_{2}-\omega_{1}\right) \frac{\Lambda_{2}+\Lambda_{1}}{\Lambda_{2}-\Lambda_{1}} .
$$

Then the map $\psi_{1}(t)=\mu \times e^{i \eta t} \psi(\lambda t)$ with $\mu>0$ has a $\rho$ pseudo support $\left[\omega_{1}, \omega_{2}\right]$ and a time domain support $\left[\frac{\Lambda_{2}-\Lambda_{1}}{\omega_{2}-\omega_{1}} L_{1}, \frac{\Lambda_{2}-\Lambda_{1}}{\omega_{2}-\omega_{1}} L_{2}\right]$.

Proof. Since $\hat{\psi}_{1}(\xi)=\mu \times \hat{\psi}\left(\frac{\xi-\eta}{\lambda}\right)$, one can deduce $\rho$ pseudo supp $\psi_{1}=\eta+\lambda \times \rho$ pseudo supp $\psi$ and then the proposition.

The different choices for the filters $\psi_{1}$ and $\psi_{2}$ are enlightened by Proposition 1.1. For computational reasons, we will use the Gabor wavelets ${ }^{2}$ defined as

$$
\psi(t)=e^{i \eta t} g(t) \quad \text { where } \quad g(t)=\frac{1}{\left(\sigma^{2} \pi\right)^{1 / 4}} e^{-\frac{t^{2}}{2 \sigma^{2}}}
$$

see for instance, [17]. This wavelet has the same time and frequency $\rho$ pseudo support $[-L, L]=[-3.5,3.5]$ with $\rho=0.9995$. In the spectral domain, we have

$$
\hat{\psi}(t)=\hat{g}(\xi-\eta), \hat{g}(\xi)=\left(4 \pi \sigma^{2}\right)^{1 / 4} e^{\frac{-\sigma^{2} \xi^{2}}{2}}
$$

By using Prop. 1.1, we can fit the Gabor wavelet inside the orthosympathetic band, respectively the parasympathetic frequency one. We obtain the two Gabor wavelets defined by (3) with the following choice of parameters:

$$
\begin{aligned}
\eta_{1} & =\frac{\omega_{1}+\omega_{2}}{2} & & \text { and } & \sigma_{1} & =\frac{2 L}{\omega_{2}-\omega_{1}} \\
\eta_{2} & =\frac{\omega_{2}+\omega_{3}}{2} & & \text { and } & \sigma_{2} & =\frac{2 L}{\omega_{3}-\omega_{2}}
\end{aligned}
$$

Moreover $\mid \rho$ pseudo Supp $\psi_{1} \mid=\frac{4 L^{2}}{\omega_{2}-\omega_{1}}$ and $\mid \rho$ pseudo Supp $\psi_{2} \mid=\frac{4 L^{2}}{\omega_{3}-\omega_{2}}$ with $\rho=0.9995$. Fig. 3 displays the Gabor wavelets coefficients in the orthosympathetic and parasympathetic bands respectively for the sample plotted in Fig.4.

\subsection{Classification and extraction of homogeneous HR profiles}

Cluster analysis has been widely studied and used in many applied areas such as medicine, chemistry, social studies and psychology. Its main purpose is to identify groups or clusters present in the data. Clustering algorithms can be divided into two main categories: hierarchical methods and partitioning methods. Hierarchical methods are stepwise and either agglomerative or divisive. For instance if $n$ objects have to be clustered, then in each step, two clusters are chosen and merged. This process continues until all objects are clustered into one

\footnotetext{
${ }^{1}$ Let $0<\rho<1$, a map $g \in L^{2}(\mathbb{R})$ and $I$ compact interval. The map $g$ have a $\rho$ pseudo support if $\frac{\int_{I}|g(t)|^{2} d t}{\int_{\mathbb{R}}|g(t)|^{2} d t}=\rho$.

${ }^{2}$ In [3] Daubechies wavelets have been investigated, they give similar results but cost more computation time. Using the Gabor wavelet is more efficient and is at least 8 times faster.
} 
group. On the other hand, divisive methods begin by putting all objects in one cluster. In each step, a cluster is chosen and split up into two. This process continues until $n$ clusters are produced. While hierarchical methods have been successfully applied to many biological applications (e.g. for producing taxonomies of animals and plants [15]), they are well known to suffer from the fact that they can not undo what was decided previously.

Principal component analysis (PCA) is a powerful tool for analyzing the correlations between several variables. It provides new uncorrelated components with higher informative power; combining the PCA with a hierarchical cluster analysis will overcome the evident strong correlation between the studied variables.

Let us denote $\Theta^{R}=\left(\theta_{1}^{R}<\theta_{2}^{R}<\cdots<\theta_{m}^{R}\right), \Theta^{L}=\left(\theta_{1}^{L}<\theta_{2}^{L}<\cdots<\theta_{n}^{L}\right)$ and $\Theta^{H}=\left(\theta_{1}^{H}<\theta_{2}^{H}<\cdots<\theta_{p}^{H}\right)$ the change points of respectively the $R R$ series, the LF and HF energies. We gather these change points sequences in $\mathcal{T}=\Theta^{R} \cup \Theta^{L} \cup \Theta^{H}$. Let us consider the ordered instants $\mathcal{T}=\tau_{1}<\tau_{2}<\cdots<\tau_{M}$ of $\mathcal{T}$ where $M \leq m+n+p$. We will be interested in the following variables:

- The variable $\theta_{i}^{R}-\theta_{i-1}^{R}$ which represents the time lapse where the $\mathrm{RR}$ signal has a stationary behaviour.

- The variable $\theta_{i}^{H}-\theta_{i-1}^{H}$ which represents the duration of the $i^{\text {th }}$ level of the HF energy. This duration can be seen as the duration where only the parasympathetic (braking) system is activated and has a fixed regime.

- The variable $\theta_{i}^{L}-\theta_{i-1}^{L}$ which represents the duration of the $i^{\text {th }}$ level of the LF energy. This duration can be seen as the lapse of time where only the orthosympathetic (acceleration) system is in action and has established a fixed regime.

- the variable $\tau_{i}-\tau_{i-1}$ which represents the inter $R R, H F$ and $L F$ durations of the $i^{\text {th }}$ level of the HF energy before one of the two systems switches to another state.

For a given individual we will construct a table that describes different states of the heart rate variability during the measurements. In this case we will have $M$ states, for each we have the durations, $\Delta \theta^{R}, \Delta \theta^{H}, \Delta \theta^{L}$ and $\Delta \tau$. On this lapse of time there is at least one change of the heart rate behaviour. This can be represented by the following table:

\begin{tabular}{|c|c|c|c|c|c|c|c|}
\hline States & $\Delta \theta^{R}$ & $\Delta \theta^{L}$ & $\Delta \theta^{H}$ & $\Delta \tau$ & $R R$ & $L F$ & $H F$ \\
\hline 1 & $\theta_{2}^{R}-\theta_{1}^{R}$ & $\theta_{2}^{L}-\theta_{1}^{L}$ & $\theta_{2}^{H}-\theta_{1}^{H}$ & $\tau_{2}-\tau_{1}$ & $R R_{1}$ & $L F_{1}$ & $H F_{1}$ \\
\hline & $\vdots$ & $\vdots$ & $\vdots$ & $\vdots$ & $\vdots$ & $\vdots$ & $\vdots$ \\
\hline M-1 & $\theta_{M}^{R}-\theta_{M-1}^{R}$ & $\theta_{M}^{L}-\theta_{M-1}^{L}$ & $\theta_{M}^{H}-\theta_{M-1}^{H}$ & $\tau_{M}-\tau_{M-1}$ & $R R_{M-1}$ & $L F_{M-1}$ & $H F_{M-1}$ \\
\hline $\mathrm{M}$ & $T-\theta_{M}^{R}$ & $T-\theta_{M}^{L}$ & $T-\theta_{M-1}^{H}$ & $T-\tau_{M}$ & $R R_{M}$ & $L F_{M}$ & $H F_{M}$ \\
\hline
\end{tabular}

Table 1: Presentation of the variables used for PCA projection and clustering.

In order to illustrate how the discriminating variables will be constructed, we give a simple example in Fig.2.

\section{REAL CASE ANALYSIS}

In this section we apply classification techniques to a real case by investigating heart rate variability of an emergency physician during a 2-hour shift at the University Hospital of Clermont-Ferrand. Among workers, emergency physicians represent a population at risk because their work is a complex interplay between the management of life-and-death emergencies, lack of sleep and fatigue. This job demands long-working hours, sustained vigilance and unpredicted stressful situations. The aim of this paper is to classify different states 


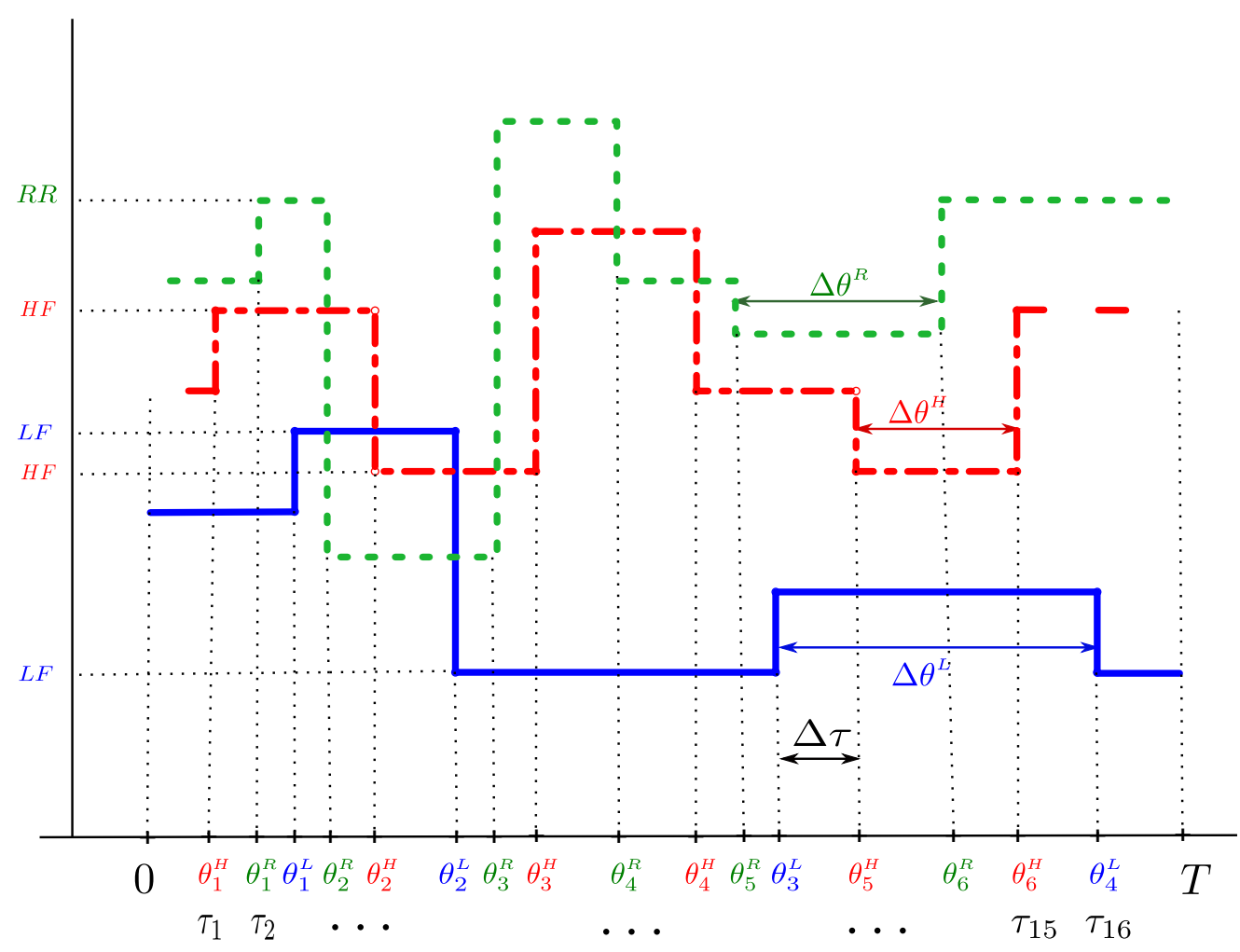

FiguRE 2. Illustration on how we construct the variables $\Delta \tau, \Delta \theta$

of HR activity during a 24-hour shift. For this purpose, we make a blind separation of groups that highlights nychthemeral cardiac behaviour. Though we dispose of larger data sets with ECG measurements, we only focus on one emergency physician to show the efficiency of our approach. A further discriminant analysis will be investigated for the whole data collected from all 19 emergency physicians' measurements. The idea is to give a theoretical framework that will be used to classify a cohort of emergency physicians.

\subsection{Data description}

This paper presents a methodological approach and can be seen as an introductory work for further study that will concern large number of individuals. Indeed, we collected a very large dataset from the 19 emergency physicians of the Clermont-Ferrand University Hospital (CHU), France. This study takes part of [11], the protocol was approved by the Regional Ethics Committee of the Clermont-Ferrand University Hospital (CHU), France. The study was explained to emergency physicians who provided their written informed consent. Exclusion criteria included endocrine disease, pregnancy, deleterious life event, any current illness, anti-inflammatory or chronotropic drugs. The ECG signal analysis was investigated on 24-hour electrocardiogram recorded from 8:30 until 8:30 the next morning. For technical testing of our approach, we will only use the ECG from one emergency physician. The ECG signal was cleaned by removing artifact measures and converted to RR signal. The data preprocessing is summarized in the following algorithm: 


Algorithm 1: Data preprocessing for the construction of table1

Input: Cleaned RR signal $X(t)$ on time interval $[0, T]$

Find $b_{0}$ and $b_{f}$ first and last possible instant for which wavelets transform can be calculated.

foreach $b=b_{0} \ldots b_{f}$ do

- Calculate the log-energies $Z_{1}(b)$ and $Z_{2}(b)$ given in (2) by using Gabor wavelets $\psi_{1}$ and $\psi_{2}$.

$\left(R R_{i}\right)$

- Apply the FDpV method on the $R R$ cleaned signal $X(t)$ to extract $\left(\theta_{i}^{R}\right)$ and the corresponding

- Apply the FDpV method to $\left(Z_{1}(b), b=b_{0} \ldots b_{f}\right)$ and extract $\left(\theta_{i}^{L}\right)$ and its $\left(L F_{i}\right)$

- Apply the FDpV method to $\left(Z_{1}(b), b=b_{0} \ldots b_{f}\right)$ to extract $\left(\theta_{i}^{H}\right)$ and the corresponding $\left(H F_{i}\right)$

Deduce the differences $\Delta \theta^{R}, \Delta \theta^{L}, \Delta \theta^{H}$

Output: Variables $\Delta \theta^{R}, \Delta \theta^{L}, \Delta \theta^{H}$, mean RR, LF and HF

The output of the last algorithm creates the table 1, in which we consider the columns as the discriminating variables of the heart activity states.

\subsection{Results and graphical representations}

At first glance, we can realize that variables, given in table 1, are correlated and should be reduced to a lower dimension. For this reason we will perform the classification on the principal components instead of the initial data. The idea is to get rid of dependence or correlation between the initial variables and benefit from their orthogonality. After the PCA has been performed we make a hierarchical classification on the resulting principal components. Fig. 3 highlights three distinct clusters reflecting the heart rate behaviour of the studied individual.

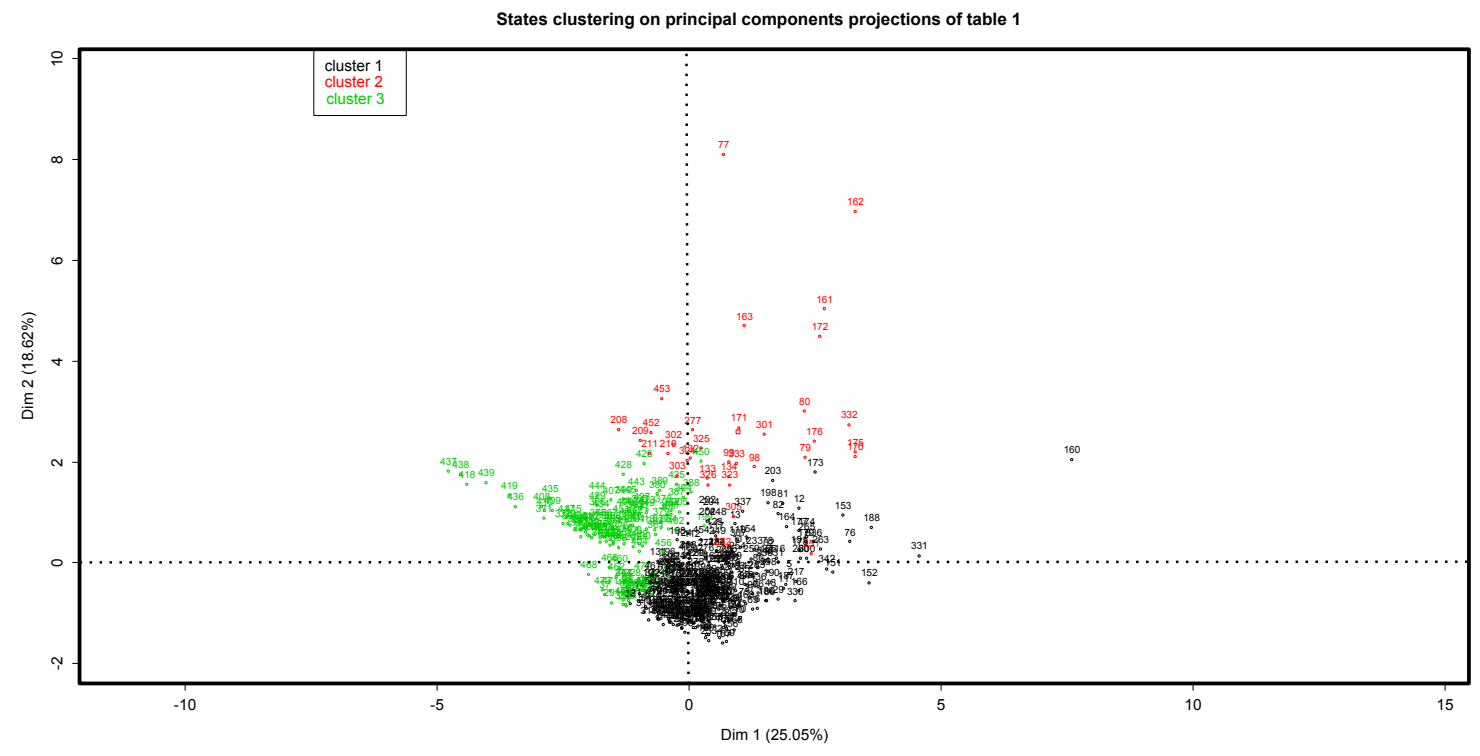

FiguRE 3. Example of the state projections on the first principal components plan 
In order to locate each cluster in the RR, HF and LF during the activity day timeline, we reproduce the mean signals and their corresponding change points. In Fig. 4, we see clearly what happens to the autonomous nervous system activity. We recognize in Fig. 4 the specific behaviour of the autonomic nervous two opposite systems(parasympathetic and orthosympathetic); it is reflected here by an alternation of the low and high frequency energies. This type of classification suggest that it would possible to extract an indicator of stress or well-being by comparing the occurrence of this clusters to the actual feeling of the physician during his shift.
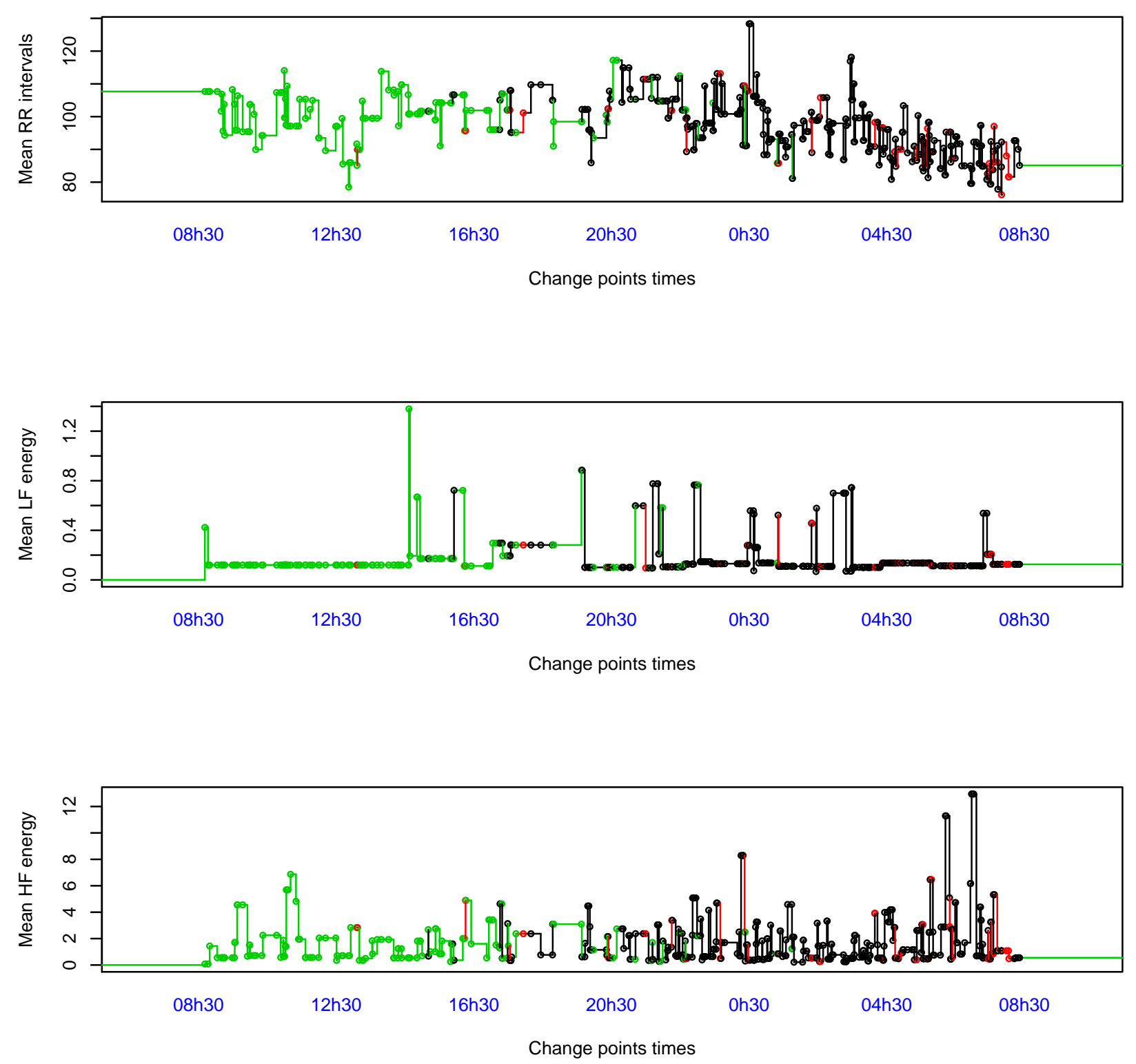

Figure 4. Example of states classification during 24-hour shift 
We can also quantify the discriminating power of each variable on the classification. Doing so, we determine what characteristics explain most cluster differentiations.

\section{Conclusions}

We introduce a methodological approach highlighting the action of the autonomous nervous system on the heart rate activity during a 24-hour shift of an emergency physician. The use of spectral analysis with Gabor wavelets permits to extract energies corresponding to high and low frequency bands. The resulting energies are used to construct discriminating variables that reflect the way heart rate is modulated by the emergency physician activity.

Further research should be conducted on a large number of individuals. The classification results will be combined with a survey concerning emergency physician real feel during their shift. This will allow to extract stress or well-being indicators.

\section{REFERENCES}

[1] A. Ayache and P. R. Bertrand, Discretization error of wavelet coefficient for fractal like processes, Advances in Pure and Applied Mathematics, 2 (2011), pp. 297-321.

[2] J.-M. BARdet AND P. Bertrand, Identification of the multiscale fractional brownian motion with biomechanical applications, Journal of Time Series Analysis, 28 (2007), pp. 1-52.

[3] J.-M. BARDEt AND P. R. Bertrand, A non-parametric estimator of the spectral density of a continuous-time gaussian process observed at random times, Scandinavian Journal of Statistics, 37 (2010), pp. 458-476.

[4] J.-M. BARDet, H. BiBI, AND A. Jouini, Adaptive wavelet-based estimator of the memory parameter for stationary gaussian processes, Bernoulli, 14 (2008), pp. 691-724.

[5] M. Basseville And I. Nikiforov, Detection of abrupt changes: Theory and application. 1993, Information and System sciences, Prentice-Hall.

[6] P. R. Bertrand, M. Fhima, And A. Guillin, Off-line detection of multiple change points by the filtered derivative with p-value method, Sequential Analysis, 30 (2011), pp. 172-207.

[7] A. Camm, M. Malik, J. Bigger, G. Breithardt, S. Cerutti, R. Cohen, P. Coumel, E. Fallen, H. Kennedy, R. Kleiger, ET AL., Heart rate variability: standards of measurement, physiological interpretation and clinical use. task force of the european society of cardiology and the north american society of pacing and electrophysiology, Circulation, 93 (1996), pp. 10431065.

[8] A. Chamoux and P. Catalina, Le système holter en pratique, Médecine du Sport, 58 (1984), pp. 43-273.

[9] M. O. Diab, C. Marque, And M. A. Khalil, Classification for uterine emg signals: Comparison between ar model and statistical classification method, INTERNATIONAL JOURNAL OF COMPUTATIONAL COGNITION (HTTP://WWW. IJCC. US), 5 (2007).

[10] F. Dutheil, G. Boudet, C. Perrier, G. Lac, L. Ouchchane, A. Chamoux, M. Duclos, and J. Schmidt, Jobstress study: Comparison of heart rate variability in emergency physicians working a 24-hour shift or a 14-hour night shift a randomized trial, International journal of cardiology, 158 (2012), pp. 322-325.

[11] F. Dutheil, M. Trousselard, C. Perrier, G. Lac, A. Chamoux, M. Duclos, G. Naughton, G. Mnatzaganian, and J. SCHMidT, Urinary interleukin-8 is a biomarker of stress in emergency physicians, especially with advancing age the jobstress* randomized trial, PloS one, 8 (2013), p. e71658.

[12] U. Frisch, Turbulence, Turbulence, by Uriel Frisch, pp. 310. ISBN 0521457130. Cambridge, UK: Cambridge University Press, January 1996., 1 (1996).

[13] A. Goldberger, Heartbeats, hormones and health : is variability the spice of life ?, Am. J. Crit. Care Med, 163 (2001), pp. $1289-1290$.

[14] P. C. Ivanov, L. A. N. Amaral, A. L. Goldberger, S. Havlin, M. G. Rosenblum, Z. R. Struzik, and H. E. Stanley, Multifractality in human heartbeat dynamics, Nature, 399 (1999), pp. 461-465.

[15] L. Kaufman and P. J. Rousseeuw, Finding groups in data: an introduction to cluster analysis, vol. 344, Wiley. com, 2009.

[16] N. Khalfa, P. R. Bertrand, G. Boudet, A. Chamoux, and V. Billat, Heart rate regulation processed through wavelet analysis and change detection: Some case studies, Acta biotheoretica, 60 (2012), pp. 109-129.

[17] S. Mallat, A wavelet tour of signal processing. 1998.

[18] G. SAmorodnitsky And M. TAQQU, Stable non-gaussian random processes: stochastic models with infinite variance. 1994, Chapman Hall, New York. 